\title{
Rating the Stock Market Performance
}

\section{through Zero-Investment Strategies}

\author{
Maria-Teresa Bosch-Badia ${ }^{1}$, Joan Montllor-Serrats ${ }^{2} \&$ Maria-Antonia Tarrazon-Rodon ${ }^{3}$ \\ ${ }^{1}$ Visiting researcher, Department of Economics, Universitat de Girona, Girona, Spain \\ ${ }^{2}$ Professor of Finance, Department of Business, Universitat Autonoma de Barcelona, Spain \\ ${ }^{3}$ Associate Professor of Finance, Department of Business, Universitat Autonoma de Barcelona, Spain \\ Correspondence: Joan Montllor-Serrats, Professor of Finance, Department of Business, Universitat Autonoma de \\ Barcelona, Spain. E-mail: joan.montllor@uab.cat
}

Received: November 16, 2014

Accepted: January 3, $2015 \quad$ Online Published: January 10, 2015

doi:10.5430/afr.v4n1p129

URL: http://dx.doi.org/10.5430/afr.v4n1p129

\begin{abstract}
The central aim of this work is to find a method for rating the performance of stock market indices. Although stock market indices are the most frequent benchmark for portfolio performance measurement, there is no benchmark for them except its own history and other market indices. Comparing the present performance with the past or with other markets means to introduce the variables time and distance into the analysis, which leads to compare different contexts. This arouses the question whether we can measure the stock market performance putting aside time and distance. We find a positive answer by means of options that have the index as their underlying asset. Properly chosen, these options create thresholds that classify the performance level on each period according to a systematic scale. To this end, we develop an option based zero-investment strategy that interacts a long call and short put that capture the market risk premium at their maturity. The notional return on this strategy can be taken as a performance measure that enables us to rate the market performance according to four categories depending on its capacity of overcoming or not the cost of protecting a long position with a put or a short position with a call. We prove that our measure adds a new interpretation to the Sharpe ratio because it is quasi-proportional to it for the usual values of volatility. The paper includes an application to the historical performance of S\&P 500 (1959-2013).
\end{abstract}

Keywords: Performance measures, Notional return, Sharpe ratio, Market indices

\section{Introduction}

The central aim of this work is to find a method for rating the performance of stock market indices. Although stock market indices are the most frequent benchmark for portfolio performance measurement, there is no benchmark for them except its own history and other market indices. Comparing the present performance with the past or with other markets means to introduce the variables time and distance into the analysis, which leads to compare different contexts. This arouses the question whether we can measure the stock market performance putting aside time and distance. We can find a positive answer by means of derivates that have the index as their underlying asset. Properly chosen, derivatives on the market index can create thresholds calculated from the features of the same index in the same period. To this end, we break the index portfolio into the addition of a risk free asset and an option based zero-investment strategy. The straightforward advantage of this approach is that it enables us to compare the risk premium with the notional value of the zero-investment strategy, which, as shown in this article, constitutes the value of the risky position of the asset under analysis. The strategy consists of combining long and short positions on call and put standard European options that capture the risk premium on the market index. The notional value of the strategy equates the value of both the upside risk and the downside risk respectively measured through the two opposite options. The importance of zero-investment strategies in performance measurement is underlined by Sharpe (1994). Although in the development of the article we apply the Black and Scholes (1973) formula, it can substituted it by other valuation formulae if the latter satisfies better the requirements of the analysis. The measure we obtain from standard options creates a benchmark that is only a function of volatility. The application of this benchmark to the performance analysis of a market index in different periods automatically assembles them into four categories according to the performance level of each period. Beyond market indices, the method we develop can be applied to any asset. The four categories in which we classify the periods or the assets under analysis differ from quartiles. The 
position of an asset in a quartile depends on the performance of the rest of assets, but the position of an asset in one of these categories only depends on its own performance. Finally, we prove that our performance measure is quasi-proportional to Sharpe ratio for the usual range of volatilities, which opens a new way to interpret it. Performance analysis has adopted the points of view of total risk, systematic risk and asymmetric risk measures according to the purposes of the study. The seminal work by Sharpe (1966) focuses on total risk from the mean-variance perspective. Modigliani and Modigliani (1997) develop MSquared in order to obtain a straightforward interpretation of the relationship between risk and return. Graham and Harvey (1997) introduce the variability or the risk free interest rate on performance measurement. The Treynor ratio (Treynor 1965) focuses on systematic risk. Jensen's alpha (Jensen 1968) shows the excess risk premium with respect to the required risk premium according to systematic risk. The Treynor-Black ratio (Treynor \& Black, 1973) consists of the quotient between alpha and specific risk. The observed asymmetries in the returns of financial assets and the growth of hedge funds have drawn the attention of performance research to asymmetric risk measures. The Sortino (Sortino \& van de Meer 1991) and Sortino-Satchell ratios (Sortino \& Satchell, 2001) measure performance with respect to downside risk. Bernardo and Ledoit (2006) opt for comparing gains and losses with the aim of overcoming the limitations of Sharpe ratio to deal with heavy tails. The Omega ratio (Shadwick \&Keating, 2002) also compares gains and losses but taking explicitly into account skewness and kurtosis. As shown in Section 4, the measure we propose can be related to the Sharpe-Omega ratio defined by Kazemi, Schneeweis, and Gupta (2004) after the Omega ratio. The Sharpe-Omega ratio has been until now applied to the performance analysis of hedge funds (Hentati, Kaffel, \& Prigent, 2010; van Dyk, van Vuuren, \& Heymans, 2014; Proelss \& Schweizer, 2014), portfolio insurance (Bertrand \& Prigent, 2011), structured products (Hentati \& Prigent, 2012), and Real Estate Investment Trusts (Gianotti \& Mattarocci 2013). Farinelli and Tiblietti (2008) generalize Omega. The Rachev ratio (Rachev, Stoyanov, \&Fabozzi 2007) belongs to the same family. The Homm -Pigorsch ratio (Homm \& Pigorsch 2012) compares the risk premium with the Aumann and Serrano (2008) risk measure, which is sensible to skewness and kurtosis Eling and Schuhmacher (2007) show the practical robustness of Sharpe ratio even when it is applied to hedge funds.

The paper is structured as follows. In Section 2 we develop the basic strategy. In Section 3 we present the concept of risk level that is central for the explanation of the risk premium. Section 4 is devoted to the analysis of the notional return on the zero investment strategy. Section 5 introduces the concepts of long and short hedging level that lead to the identification of the different rating levels presented in section 6. Section 7 enlarges the previous analysis by developing the composition ratio that links the risk level with the notional return. In Section 8 we show that the notional return adds a new meaning to the Sharpe ratio through the quasi proportionality property we prove. In Section 9 we apply the previous theoretical developments to the analysis of the behaviour of S\&P 500 between 1959 and 2013. Section 10 concludes the article.

\section{Building up the Zero-Investment Strategy}

The risk premium on any asset can be expressed as the outcome of a zero-investment strategy. Sharpe (1994) defines a zero-investment strategy as any strategy that involves a zero outlay of money at its inception and returns either a positive, negative or zero amount in the future. Any zero investment strategy consists of a long and a short positions that are equal one each other. This value, i. e. the value of the long and the short position, is the notional value of the zero investment strategy. Be a zero-investment strategy at one period horizon constituted by a long European call plus a short European put, both with a strike price equal to the asset price at the beginning of the period $\left(\mathrm{A}_{0}\right)$ capitalized at the risk free interest rate (r). These positions create a zero-investment strategy because, as we argue after equation (4), the values of both options are equal. An investor who holds them captures the asset risk premium at the end of the period. Denoting by $c_{1}$ the call value at the end of the period, by $p_{1}$ the value of its corresponding put, and by $\mathrm{A}_{1}$ the final value of the asset, we can write the final option values as:

$$
\begin{aligned}
& c_{1}=\operatorname{Max}\left[A_{1}-A_{0} \cdot(1+r), 0\right] \\
& p_{1}=\operatorname{Max}\left[A_{0} \cdot(1+r)-A_{1}, 0\right]
\end{aligned}
$$

Their algebraic sum, i.e. long call minus short put, is the final value of the zero-investment strategy:

$$
c_{1}-p_{1}=A_{1}-A_{0} \cdot(1+r)
$$

Be $R_{A}$ the rate of return on the asset under analysis. Substituting $A_{1}$ in (3) by $A_{0}$ capitalized at $R_{A}$, the final value of the strategy can be written as:

$$
c_{1}-p_{1}=A_{0} \cdot\left(R_{A}-r\right)
$$

which shows that the outcome of this zero-investment strategy at the end of the period is the asset risk premium 
expressed in monetary units.

It stems from the put-call parity relationship, and from the equality between the present values of the asset and the strike price in this strategy, that the put and call have the same value at the beginning of the period, which is at the same time the notional value of the strategy $\left(n t_{0}\right)$ :

$$
c_{0}=p_{0}=n t_{0}
$$

The call captures the upside risk of the asset, while the put captures its downside risk. Thus, the notional value of the strategy can be regarded as the value of the risk embedded in the asset. A zero-investment strategy always consists of a long position compensated by a short position. The peculiarity of the put-call strategy is that its positions measure the value of the opportunity of turning into profits the upside risk through the call and the downside risk through the put. This strategy can be applied to any asset, but its differential feature is that it can be applied to the analysis of the benchmark's performance, as we argue in Section 6 where we show its specific merits for the performance analysis of market indices. In fact, to measure performance through the notional return, traded options are not needed. Only market data that enable us to calculate options' values are needed.

\section{The Risk Level}

Sharpe (1994) defines the relative position of a zero-investment strategy as the ratio of the notional value of this strategy on the value of the investor's assets. Centring this concept on the asset under analysis, we define the risk level as the quotient between the notional value of the zero-investment strategy and the asset. We designate it by $\gamma$ :

$$
\gamma_{A}=\frac{n t_{0}}{A_{0}}
$$

Then, the risk level can be interpreted as the monetary units of the value of the risk embedded in the asset $\left(n t_{0}\right)$ per monetary value of the asset itself.

It stems from (5) and (6) that the risk level can be valued as either a call or a put divided by the asset present value that, at the same time, equals the present value of the strike price. Applying the Black and Scholes formula, we obtain that the risk level is exclusively a function of volatility (Note 1):

$$
\gamma_{A}=2 \cdot N\left(\frac{\sigma_{A}}{2}\right)-1
$$

The study of this formula shows that the risk premium is a monotone increasing function of volatility. The evolution of the risk level according to volatility is depicted in Figure 1. This figure, shows, in addition, the evolution of the equally weighted level that we mention in Section 7.

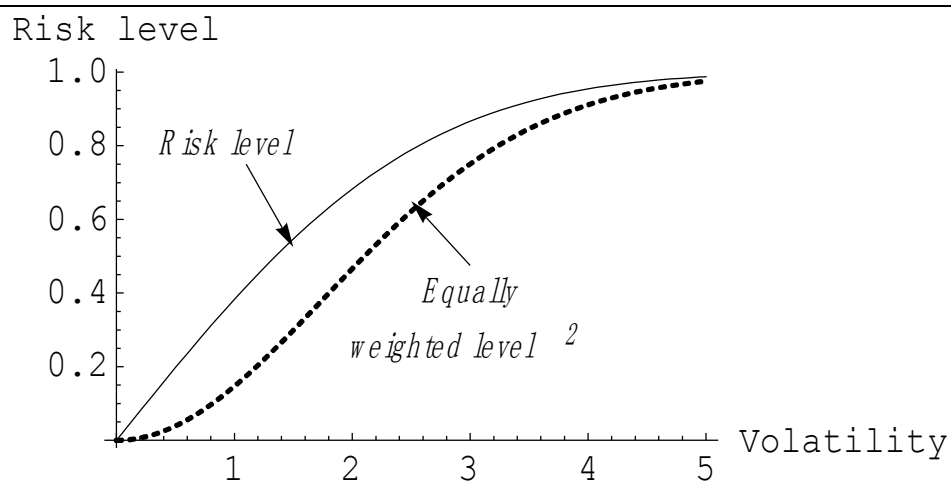

The risk level is a concave function with an almost constant slope for the usual range of volatilities (i.e. volatilities under 50\%). The equally weighted level evolves from a convex to a concave function. For very high volatilities, both functions converge to a horizontal asymptote equal to 1 .

Figure 1. Risk Level and Equally Weighted Level

Since volatility can take values between zero and infinity, the corresponding values of the risk level, according to (7), go between zero and one:

$$
0 \leq \gamma_{A} \leq 1
$$




\section{The Notional Return on the Zero-Investment Strategy}

The notional return on the zero-investment strategy consists of the algebraic addition of the returns on the long call and the short put. The former $\left(y_{c}\right)$ is:

$$
y_{c}=\frac{\operatorname{Max}\left[A_{0} \cdot\left(R_{A}-r\right), 0\right]}{c_{0}}-c_{0}
$$

while the rate of return on the short put is $\left(y_{p}\right)$ :

$$
y_{p}=p_{0}-\frac{\operatorname{Max}\left[A_{0} \cdot\left(r-R_{A}\right), 0\right]}{p_{0}}
$$

Then, the rate of return on the strategy $\left(\zeta_{A}\right)$, that we call notional return, can be written as:

$$
\zeta_{A}=\left(\frac{\operatorname{Max}\left[A_{0} \cdot\left(R_{A}-r\right), 0\right]}{c_{0}}-c_{0}\right)-\left(\frac{\operatorname{Max}\left[A_{0} \cdot\left(r-R_{A}\right), 0\right]}{p_{0}}-p_{0}\right)
$$

Taking into account that, as seen in (5), the initial values of the call and the put are equal one to each other, substituting (5) in (11), after some algebraic operations we obtain that the notional return turns out to be:

$$
\zeta_{A}=\frac{A_{0} \cdot\left(R_{A}-r\right)}{n t_{0}}
$$

Thus, the rate of return on the zero-investment strategy or notional return equates the ratio between the asset risk premium and the notional value of the strategy. Substituting (6) into this result, the rate of return on the zero-investment strategy becomes the ratio between risk premium and risk level:

$$
\zeta_{A}=\frac{R_{A}-r}{\gamma_{A}}
$$

Then we can express risk premium as the product of risk level per notional return:

$$
R_{A}-r=\gamma_{A} \zeta_{A}
$$

The notional return we have obtained can be related to the Sharpe-Omega ratio defined by Kazemi et al. (2004). The numerator of this ratio consists of the difference between the expected return and a threshold previously chosen. Its denominator is a put on the asset under analysis with a strike price equal to the threshold. As these authors point out, measuring performance by means of option theory enables to capture the effects of skewness and kurtosis by switching to the appropriate valuation formula (Note 2). Equating to 1 the initial value of the asset and choosing a threshold (strike price) equal to one plus the risk free interest rate, the notional return on the zero investment strategy we have defined and the Sharpe-Omega ratio are equivalent.

\section{Long Hedging vs. Short Hedging Levels}

As it stems from (13) when the notional return equates 1 the risk premium pays back the initial value of the put:

$$
\zeta_{A}=1 \Rightarrow\left(R_{A}-r\right)^{*}=\gamma_{A}
$$

For a notional return greater than 1 , the risk premium not only pays back the put value, but also generates additional earnings. On this basis, we can say that if the notional return becomes equal to 1 the zero investment strategy reaches its long hedging level because for any greater risk premium a long position in the asset hedged with a put produces earnings. Thus, taking (7) into account, the minimum risk premium to reach the long hedging level, that we designate by $\left(R_{A}-r\right)^{*}$, is:

$$
\left(R_{A}-r\right)^{*}=2 \cdot N\left(\frac{\sigma_{A}}{2}\right)-1
$$

The long hedging level has a parallel with the short hedging level that we introduce next. For any notional return equal to -1 the risk premium of a short position in the asset pays back the cost of having hedged this short position with a call: 


$$
\zeta_{A}=-1 \Rightarrow\left(r-R_{A}\right)^{*}=\gamma_{A}
$$

Thus, for any notional return equal to or lower than -1 , the zero investment strategy reaches its short hedging level. Table 1 summarizes the different scenarios of the notional return at the maturity of the strategy and compares them with the risk premia of long and short positions.

Table 1. Notional Return vs. Risk Premium

\begin{tabular}{ccccc}
\hline Notional return & $\zeta<-1$ & $-1<\zeta<0$ & $0<\zeta<1$ & $\zeta>1$ \\
Long position & $R_{A}-r<0$ & $R_{A}-r<0$ & $0<\left(R_{A}-r\right)<\gamma$ & $\left(R_{A}-r\right)>\gamma$ \\
Short position & $r-R_{A}>\gamma$ & $0<r-R_{A}<\gamma$ & $r-R_{A}<0$ & $r-R_{A}<0$ \\
\hline
\end{tabular}

\section{Benchmark and Qualitative Classification}

After these results, we propose to measure the performance of any asset through the ratio $\left(p f_{\text {ZIS }}\right)$ between its actual risk premium and the risk premium that equates the notional value of its corresponding zero investment strategy:

$$
p f_{\text {ZIS }}=\frac{R_{A}-r}{\left(R_{A}-r\right)^{*}}
$$

Substituting the numerator of this equation according to (14), i.e. by the product $\left(\gamma_{A} \zeta_{A}\right)$, and its denominator by $\gamma_{A}$ as it follows from (15), we obtain:

$$
p f_{Z I S_{A}}=\zeta_{A}
$$

Therefore, taking into account the results summarized in Table 1, we can introduce a twofold benchmark to classify the performance of the zero investment strategy and its corresponding underlying asset. For the zero investment strategy the benchmark consists of the pair of values $\{+1,-1\}$ that determine the position of the strategy with respect to its hedging levels. From the viewpoint of the underlying asset these limits correspond to the pair of values $\left(R_{A}-r\right)^{+}$and $\left(R_{A}-r\right)^{-}$:

$$
\begin{aligned}
& \left(R_{A}-r\right)^{+}=2 \cdot N\left(\frac{\sigma_{A}}{2}\right)-1 \\
& \left(R_{A}-r\right)^{-}=1-2 \cdot N\left(\frac{\sigma_{A}}{2}\right)
\end{aligned}
$$

On this basis, we classify the performance expressed by the notional return in four categories:

1 Long hedging: A long position protected with a put would have produced profits or a nil result. In this case, the notional return is equal or greater than $1\left(\zeta_{A} \geq 1\right)$, i.e. it equates or overcomes its long hedging level. The risk premium zone is:

$$
\left(R_{A}-r\right) \geq\left(R_{A}-r\right)^{+}
$$

2 Long: An unprotected long position would have produced profits or a nil result, but, if protected with a put, it would have produced loses. The notional return is positive, but lower than $1 \quad\left(0 \leq \zeta_{A} \leq 1\right)$. The risk premium zone is:

$$
0 \leq\left(R_{A}-r\right)<\left(R_{A}-r\right)^{+}
$$

3 Short: An unprotected short position would have produced profits, but, if protected with a call, it would have produced loses. The notional return is negative, but greater than $-1\left(-1<\zeta_{A}<0\right)$. The risk premium zone is:

$$
\left(R_{A}-r\right)^{-} \leq\left(R_{A}-r\right)<0
$$

4 Short hedging: A short position protected with a call would have produced profits or a nil result. The notional return is equal or lower than $-1 \quad\left(\zeta_{A} \leq-1\right)$, i.e. it becomes equal to its short hedging level or lower than it. The risk premium zone is:

$$
\left(R_{A}-r\right)<\left(R_{A}-r\right)
$$


Positive notional returns are obtained by interlocking the exercise of the long call and the no exercise of the short put. The return on the latter $(+1)$ compensates the impact of the cost of the former $(-1)$ on the strategy return. Then, notional return equates the gross return on the call. If it is greater than 1, namely it belongs to the long hedging category, the call by itself would have produced profits. For negative notional returns, all these positions and signs are reversed. All in all, these four categories create a rating scale for assets, funds and markets performance that we can call hedging scale. The classification of an asset into the hedging scale depends only on its own achievements, i.e. in the position of its notional return with respect to the limits $\{+1,-1\}$. The benchmark does not consist now in another asset or in another period. For this reason, we can say that the rating scale we have obtained is appropriate for stock market indices because it fulfills the requirements of independence from time and distance. Certainly, these considerations do not discard the comparison between the present with the past performances of stock indices and with other stock indices. Nevertheless, since the market index is the basic benchmark to which any asset in the market is compared, it seems appropriate to enlighten the market performance by rating it on basis of its own properties. In this line, the classification of the performance of a stock index or any asset according to the hedging scale adds a qualitative indicator to the analysis of how successfully the value generating capacity embedded in its own risk level has been turned into notional return and risk premium. Benchmarking the benchmark, as the qualitative classification does, can be regarded as of particular interest for passive investors, focused by nature on the long run, for whom the history of the qualitative market performance can be more informative than the numerical series of a quantitative performance measure. The question than the notional return enables us to answer in this respect is: Does a superior category (long hedging or long) predominate in the long run? As we will show in Section 9, the answer is "yes". The same approach applied to actively managed portfolios could be regarded as less interesting because the investment strategies applied to these portfolios may have changed even in the short run. Then, any long run performance analysis informs on the management but not on the techniques.

Comparing the notional return with the Sharpe ratio as performance measures, both share the feature of expressing the risk premium per unit of risk (volatility for Sharpe ratio and risk level for notional return). Nevertheless, the notional return has the additional advantage or placing each risk premium into its corresponding qualitative category. In fact, the notional return cannot be regarded as a substitute of the Sharpe ratio, but as a complement of it. In Section 8 we explore the numerical equivalences between the notional return and the Sharpe ratio.

\section{The Weights of Risk Level and Notional Return in Risk Premium: The Composition Ratio}

The risk premium, as seen in (14), can be expressed as the product between the notional return and the risk level. The latter, that never can be negative, systematically makes a positive contribution to the risk premium. The notional return can increase, reduce or not modify the contribution of the risk level to the risk premium. Three scenarios are possible:

a. Equally weighted risk premium:

$$
\zeta=\gamma \Rightarrow(R-r)=\gamma^{2}
$$

That we designate by $(R-r)_{w}$ and can also be written as:

$$
(R-r)_{w}=\left[2 \cdot N\left(\frac{\sigma_{A}}{2}\right)-1\right]^{2}
$$

b. Notional return dominated risk premium:

$$
\zeta>\gamma \Rightarrow(R-r)>\gamma^{2}
$$

c. Risk level dominated risk premium:

$$
\zeta<\gamma \Rightarrow(R-r)<\gamma^{2}
$$

The equality between the risk level and the notional return takes place at the crossing point of their functions. It is immediate to justify that this point is unique: The risk level is a monotone increasing function of volatility that equates 0 when volatility also equates 0 , and approaches 1 when volatility approaches infinity. The notional return is a monotone decreasing function of volatility that approaches infinity when volatility approaches 0 , and approaches the risk premium when volatility approaches infinity. Figure 1, shown in Section 2, depicts the evolution of the risk level and the equally weighted level according to volatility. Figure 2 shows the functions of the risk premium level and the notional return for a risk premium of $1 \%$. At the crossing point, we can say that risk premium generation is equally weighted because both the risk level and the notional return have the same impact on risk premium value, i.e. $\sqrt{R_{A}-r}$. At its left hand side risk premium generation is return based, and at its right hand side it is risk based. 


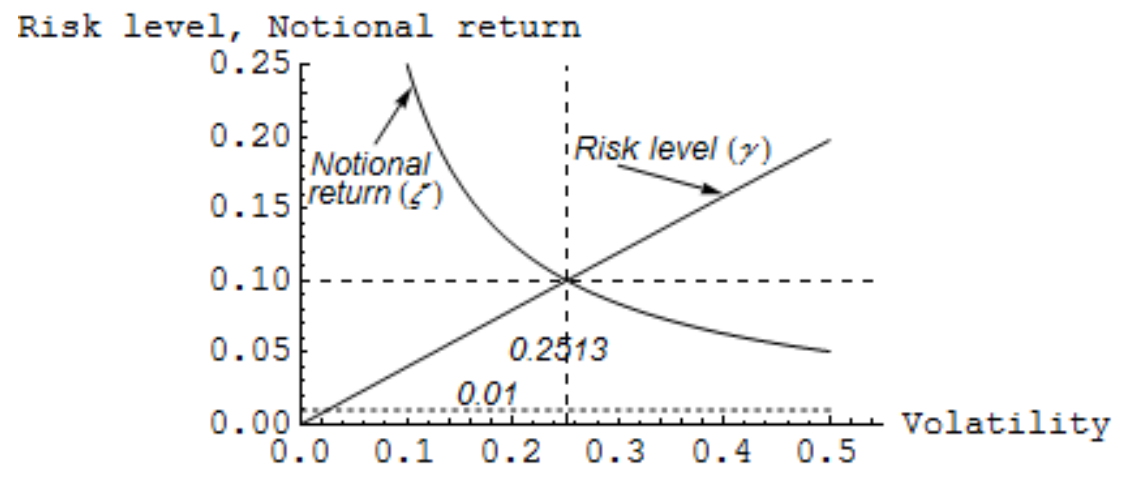

For a standard deviation of $25.13 \%$ and a risk premium of $1 \%$, the risk level becomes equal to the square root of the risk premium (10\%). Then, the notional return becomes equal to $10 \%$ as well. The shape of the risk level, although it is a strictly concave function, for the usual range of volatilities shows an almost constant slope, i.e. it is practically a straight line.

Figure 2. The Interaction between Risk Level and Notional Return

In order to analyse these scenarios, we define as the composition ratio $(w)$ the quotient that expresses how many times the risk premium contains its equally weighted value:

$$
w_{A}=\frac{R_{A}-r}{\left(R_{A}-r\right)_{w}}
$$

Recalling (14), the composition ratio can also be regarded as the number of times that the notional return contains the risk level:

$$
w_{A}=\frac{\zeta_{A}}{\gamma_{A}}
$$

From the viewpoint of the twofold impact of the notional return and the risk level in the risk premium, we can regard the composition ratio as an efficiency measure. In effect, the risk level, as shown in (14), makes by itself a positive contribution to the risk premium. If the notional return exactly makes the same contribution, the risk premium is then $\left(R_{A}-r\right)_{w}$. The composition ratio, as expressed in (30) and (31) shows to which extent the contribution of the notional return to the risk premium overperforms or underperforms the one of the risk level. An efficient behaviour of the notional return in market indices or an efficient management of it in managed portfolios can be associated to a composition ratio greater than 1 because in this case the notional return leads to a risk premium greater than the one of the equally weighted level.

Summarizing, we can say that through the zero-investment strategy we have found two reference rates for the risk premium: the hedging level and the equally weighted level. The former is the breakeven point for covering the cost of hedging the strategy. The latter indicates the risk-return equally weighted level above which the notional return predominates over the risk level in the risk premium. Volatility is the unique independent variable that determines the values of both rates.

\section{Notional Return versus Sharpe Ratio}

The Sharpe ratio and notional return are quasi-proportional for the usual range of volatilities (5\% - 50\%), as we show in the appendix. The product between the Sharpe ratio and the proportionality constant, the value of which is 2.5165 , approximately equates the notional return. Therefore, the notional return is approximately equal to 2.5 times the Sharpe ratio $(S)$. The relationship we have obtained is:

$$
2.51625 S \approx \zeta
$$

Hence:

$$
S \approx 0.397417 \zeta
$$

More specifically, for volatilities between $5 \%$ and $50 \%$ the quotient between the notional value and the Sharpe ratio is confined into the interval [1.0037, 0.9935], while for volatilities between $1 \%$ and $100 \%$, the limits of this quotient 
are [1.0136, 0.9702]. Table 2 shows the values of the constant for different volatility ranges and Figure 3 shows the conjoint evolution of Sharpe ratio and notional return.

Table 2. The Quasi-Proportionality Constant

\begin{tabular}{|c|c|c|c|c|c|c|}
\hline \multicolumn{3}{|c|}{ Volatility range } & Risk level & Relative risk level & Quasi proportionality & Product $k \cdot \ell$ \\
\hline \multirow{2}{*}{\multicolumn{2}{|c|}{$\sigma \min$}} & 0.05 & 0.0199 & 0.3989 & & 1.0037 \\
\hline & & & & & 2.51625 & \\
\hline$\sigma$ & $\max$ & 0.50 & 0.1974 & 0.3948 & & 0.9935 \\
\hline \multirow[t]{2}{*}{$\sigma$} & $\min$ & 0.0001 & 0.0000 & 0.3989 & & 1.0136 \\
\hline & & & & & 2.54077 & \\
\hline$\sigma$ & $\max$ & 1 & 0.3829 & 0.3829 & & 0.9729 \\
\hline
\end{tabular}

Here we show the calculations related to the quasi-proportionality property for the relationship between Sharpe ratio and notional return for two volatility ranges: $5 \%-50 \%$ and $0.01 \%-100 \%$. The constants have been calculated according to equation (42). The last column shows the values of the product between the constant and the relative risk level for the extremes of each range. Due to the fact that the relative risk level is a monotone decreasing function of volatility, the highest overestimation is obtained for the lowest volatility and the lowest underestimation for the highest volatility.

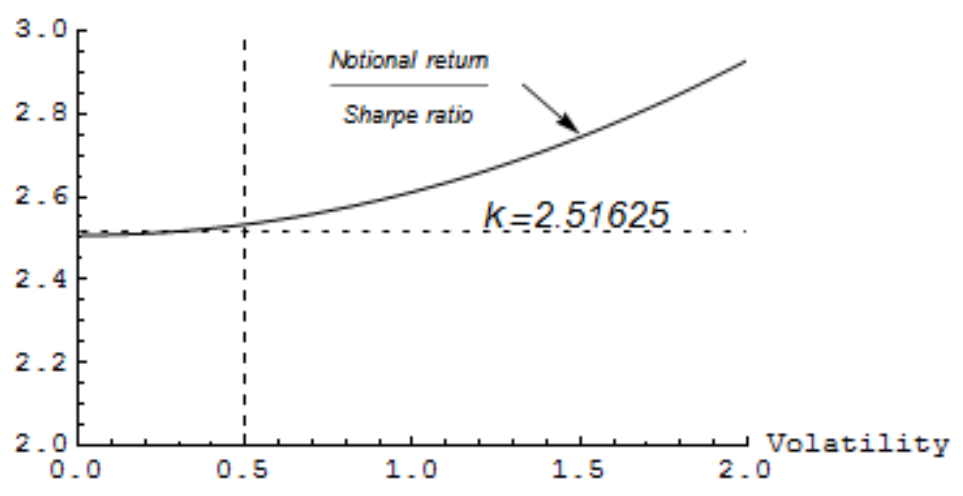

For volatilities under 0.5 , the ratio between the notional return and the Sharpe is practically equal to 2.51625. Thus, for volatilities between 0 and 0.5 the notional return and the Sharpe ratio are quasi-proportional.

Figure 3. The Quasi-Proportionality between the Notional Return and the Sharpe Ratio

\section{Empirical Application: The Performance of S\&P between 1959 and 2013}

As stated, a feature of notional return as a performance measure is that it can be applied to the market index. Substituting the market volatility in (16), we obtain the value of the long hedging benchmark for the market index. Dividing the market risk premium by this value, we obtain, according to (18) and (19), the notional return. Then, the performance of the index into the period under analysis is automatically classified in one of the four categories we have proposed. Next, we apply this procedure to the analysis of S\&P 500 performance between 1959 and 2013. Tables 3 and 4 display this analysis and Figure 4 illustrates it. 
Table 3. Notional Returns on S\&P 500 between 1959 and 2013

\begin{tabular}{|c|c|c|c|c|c|c|c|c|c|c|c|c|c|c|c|c|c|}
\hline Year & $\begin{array}{c}\text { Risk } \\
\text { premium }\end{array}$ & Volati & $\begin{array}{c}\text { Sharpe } \\
\text { ratio }\end{array}$ & & Notiona & Performance cates & $\begin{array}{c}\text { Equally } \\
\text { Weighted level }\end{array}$ & $\begin{array}{l}\text { Composition } \\
\text { ratio }\end{array}$ & ${ }^{n} \mathrm{Y}$ & Risk $\mathrm{p}$ & nVolatil & $\begin{array}{l}\text { y Sharpe }_{\text {ratio }} \\
\text { res }\end{array}$ & $R$ isk & INotiona & Perfor & hted level & $\begin{array}{c}\text { Composition } \\
\text { ratio }\end{array}$ \\
\hline & & $\sigma$ & & $\gamma$ & $\zeta$ & & $\gamma^{2}$ & $w$ & & & $\sigma$ & & $\gamma$ & $\zeta$ & & $\gamma^{2}$ & $w$ \\
\hline 1959 & 0.0952 & 0.0968 & 0.9833 & 0.0386 & 2.4657 & Long hedging & 0.0015 & 63.8852 & 1987 & 0.1612 & 0.2730 & 0.5905 & 0.1086 & 1.4848 & Long hedging & 0.0118 & 5.9785 \\
\hline 1960 & 0.0586 & 0.1100 & 0.5328 & 0.0438 & 1.3361 & Long hedging & 0.0019 & 30.4730 & 1988 & 0.0329 & 0.1863 & 0.1767 & 0.0742 & 0.4437 & Long & 0.0055 & 52.0661 \\
\hline 1961 & 0.1336 & 0.0995 & 1.3431 & 0.0397 & 3.3681 & Long hedging & 0.0016 & 84.8861 & 1989 & 0.1719 & 0.1442 & 1.1925 & 0.0575 & 2.9916 & Long hedging & 0.0033 & -20.4329 \\
\hline 1962 & -0.0230 & 0.1637 & -0.1404 & 0.0652 & -0.3524 & Short & 0.0043 & -5.4017 & 1990 & -0.0873 & 0.1640 & -0.5322 & 0.0654 & -1.3355 & Short hedging & 0.0043 & 40.7901 \\
\hline 1963 & 0.1190 & 0.0829 & 1.4355 & 0.0331 & 3.5993 & Long hedging & 0.0011 & 108.8661 & 1991 & 0.1435 & 0.1488 & 0.9643 & 0.0593 & 2.4193 & Long hedging & 0.0035 & 3.4917 \\
\hline 1964 & 0.0957 & 0.0585 & 1.6372 & 0.0233 & 4.1044 & Long hedging & 0.0005 & 175.9717 & 1992 & 0.0061 & 0.1050 & 0.0583 & 0.0419 & 0.1462 & Long & 0.0018 & 13.5265 \\
\hline 1965 & 0.0934 & 0.0744 & 1.2552 & 0.0297 & 3.1470 & Long hedging & 0.0009 & 106.0798 & 1993 & 0.0165 & 0.0875 & 0.1883 & 0.0349 & 0.4722 & Long & 0.0012 & -12.8185 \\
\hline 1966 & -0.0309 & 0.1174 & -0.2633 & 0.0468 & -0.6605 & Short & 0.0022 & -14.1160 & 1994 & -0.0241 & 0.1087 & -0.2215 & 0.0433 & -0.5556 & Short & 0.0019 & 161.1311 \\
\hline 1967 & 0.1713 & 0.0875 & 1.9586 & 0.0349 & 4.9111 & Long hedging & 0.0012 & 140.7613 & 1995 & 0.1775 & 0.0832 & 2.1332 & 0.0332 & 5.3486 & Long hedging & 0.0011 & 47.5207 \\
\hline 1968 & 0.0824 & 0.0866 & 0.9523 & 0.0345 & 2.3878 & Long hedging & 0.0012 & 69.1526 & 1996 & 0.1128 & 0.1222 & 0.9231 & 0.0487 & 2.3152 & Long hedging & 0.0024 & 24.7336 \\
\hline 1969 & -0.0008 & 0.1040 & -0.0075 & 0.0415 & -0.0189 & Short & 0.0017 & -0.4565 & 1997 & 0.1305 & 0.1823 & 0.7158 & 0.0726 & 1.7966 & Long hedging & 0.0053 & 20.2303 \\
\hline 1970 & 0.0489 & 0.1606 & 0.3043 & 0.0640 & 0.7635 & Long & 0.0041 & 11.9333 & 1998 & 0.1370 & 0.2067 & 0.6630 & 0.0823 & 1.6649 & Long hedging & 0.0068 & 15.0555 \\
\hline 1971 & 0.0782 & 0.1014 & 0.7716 & 0.0404 & 1.9349 & Long hedging & 0.0016 & 47.8545 & 1999 & 0.0978 & 0.2024 & 0.4833 & 0.0806 & 1.2135 & Long hedging & 0.0065 & -12.4872 \\
\hline 1972 & 0.1377 & 0.0833 & 1.6529 & 0.0332 & 4.1443 & Long hedging & 0.0011 & 124.7163 & 2000 & -0.1212 & 0.2476 & -0.4895 & 0.0985 & -1.2302 & Short hedging & 0.0097 & -3.5950 \\
\hline 1973 & -0.0364 & 0.1658 & -0.2194 & 0.0661 & -0.5505 & Short & 0.0044 & -8.3339 & 2001 & -0.0294 & 0.2271 & -0.1294 & 0.0904 & -0.3250 & Short & 0.0082 & -9.9778 \\
\hline 1974 & -0.1540 & 0.2338 & -0.6588 & 0.0931 & -1.6552 & Short hedging & 0.0087 & -17.7881 & 2002 & -0.1183 & 0.2738 & -0.4321 & 0.1089 & -1.0864 & Short hedging & 0.0119 & 25.6296 \\
\hline 1975 & 0.1633 & 0.1630 & 1.0022 & 0.0649 & 2.5149 & Long hedging & 0.0042 & 38.7285 & 2003 & 0.1124 & 0.1662 & 0.6764 & 0.0662 & 1.6973 & Long hedging & 0.0044 & 20.8205 \\
\hline 1976 & 0.0949 & 0.1179 & 0.8052 & 0.0470 & 2.0194 & Long hedging & 0.0022 & 42.9524 & 2004 & 0.0477 & 0.1200 & 0.3972 & 0.0479 & 0.9964 & Long & 0.0023 & -2.0522 \\
\hline 1977 & -0.0624 & 0.1004 & -0.6219 & 0.0400 & -1.5596 & Short hedging & 0.0016 & -38.9544 & 2005 & -0.0042 & 0.1128 & -0.0368 & 0.0450 & -0.0923 & Short & 0.0020 & 48.5285 \\
\hline 1978 & 0.0224 & 0.1324 & 0.1690 & 0.0528 & 0.4241 & Long & 0.0028 & 8.0317 & 2006 & 0.0886 & 0.1071 & 0.8267 & 0.0427 & 2.0733 & Long hedging & 0.0018 & 5.3964 \\
\hline 1979 & 0.0897 & 0.1140 & 0.7868 & 0.0454 & 1.9733 & Long hedging & 0.0021 & 43.4248 & 2007 & 0.0278 & 0.1802 & 0.1543 & 0.0718 & 0.3874 & Long & 0.0052 & -8.9833 \\
\hline 1980 & 0.2096 & 0.1669 & 1.2560 & 0.0665 & 3.1519 & Long hedging & 0.0044 & 47.4049 & 2008 & -0.2564 & 0.4267 & -0.6009 & 0.1689 & -1.5177 & Short hedging & 0.0285 & 16.6240 \\
\hline 1981 & -0.0373 & 0.1370 & -0.2725 & 0.0546 & -0.6835 & Short & 0.0030 & -12.5128 & 2009 & 0.1882 & 0.2675 & 0.7036 & 0.1064 & 1.7688 & Long hedging & 0.0113 & 2.6656 \\
\hline 1982 & 0.1207 & 0.1889 & 0.6387 & 0.0753 & 1.6034 & Long hedging & 0.0057 & 21.3033 & 2010 & 0.0169 & 0.1999 & 0.0845 & 0.0796 & 0.2122 & Long & 0.0063 & 30.2917 \\
\hline 1983 & 0.1242 & 0.1416 & 0.8771 & 0.0564 & 2.2004 & Long hedging & 0.0032 & 38.9942 & 2011 & 0.0409 & 0.2466 & 0.1658 & 0.0981 & 0.4168 & Long & 0.0096 & 4.25 \\
\hline 1984 & 0.0492 & 0.1395 & 0.3526 & 0.0556 & 0.8846 & Long & 0.0031 & 15.9029 & 2012 & 0.0646 & 0.1410 & 0.4586 & 0.0562 & 1.1504 & Long hedging & 0.0032 & 20.47 \\
\hline $\begin{array}{l}1985 \\
1986\end{array}$ & $\begin{array}{l}0.1602 \\
0.1204\end{array}$ & $\begin{array}{l}0.1058 \\
0.1582\end{array}$ & $\begin{array}{l}1.5146 \\
0.7612\end{array}$ & $\begin{array}{l}0.0422 \\
0.0631\end{array}$ & $\begin{array}{l}3.7984 \\
1.9101\end{array}$ & $\begin{array}{l}\text { Long hedging } \\
\text { Long hedging }\end{array}$ & $\begin{array}{l}0.0018 \\
0.0040\end{array}$ & $\begin{array}{l}30.2917 \\
13.6781\end{array}$ & 2013 & 0.1824 & 0.1190 & 1.5324 & 0.0475 & 3.8434 & Long hedging & 0.0023 & 80.97 \\
\hline
\end{tabular}

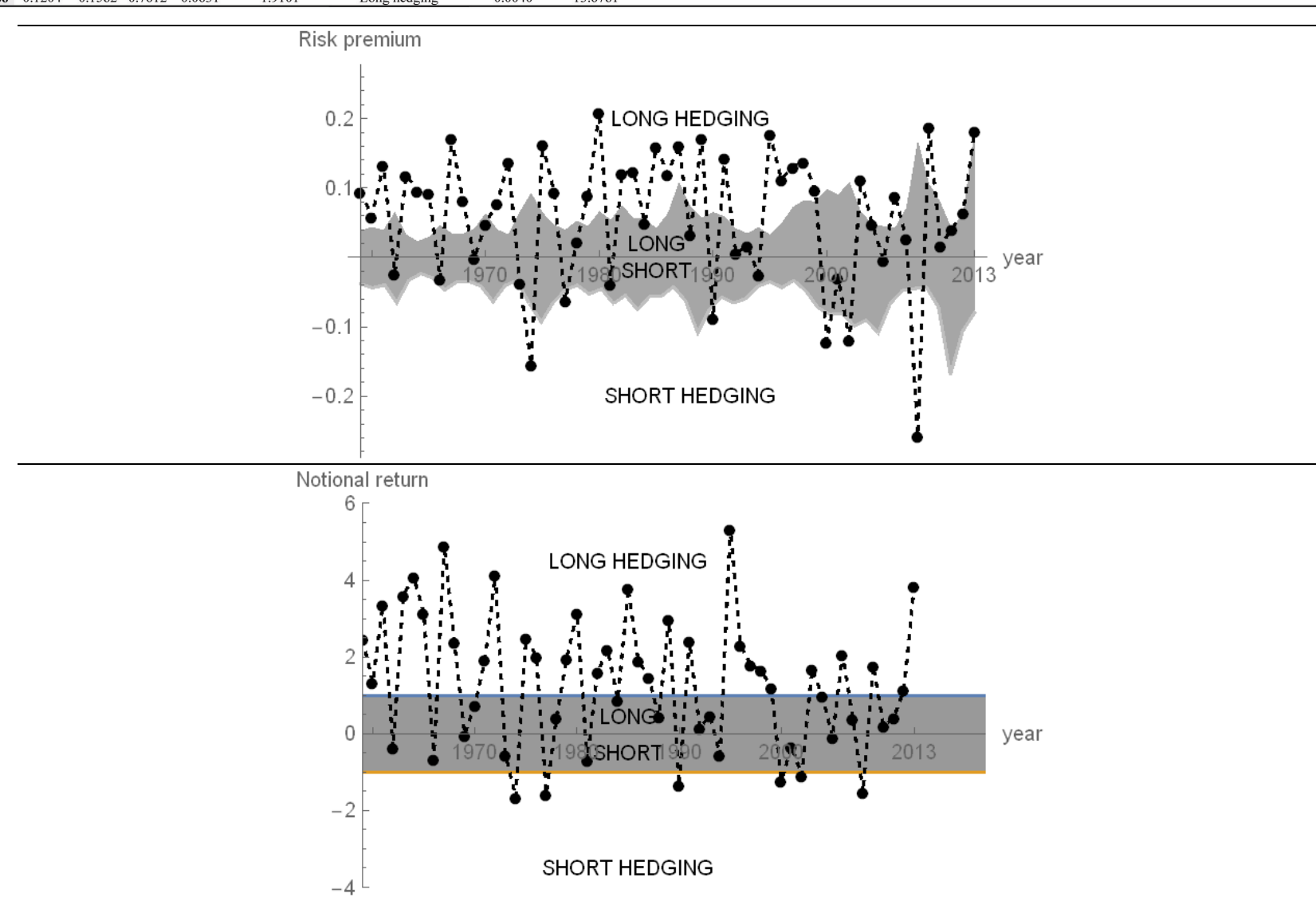

The upper plot shows the evolution of risk premia with respect to their hedging levels according to the data shown in Table 3. The dots stand for the values of risk premia. The shadowed zone marks the limits of long and short categories. Dots over the shadowed zone belong to the long hedging category, while the ones under it belong to the short hedging category. The bottom plot parallels the upper plot, stating the different categories in respect to notional returns instead of risk premium. Thus, as said in the text, the hedging limits become +1 and -1 .

Figure 4. S\&P 500 between 1959 and 2013 Risk Premium, Notional Return, and Performance Categories 
Table 3 applies the results of Sections 2-7 to the study of the evolution of S\&P 500 between 1959 and 2013. The risk premium and its standard deviation for each year are calculated from the daily adjusted values of the S\&P 500 and the 6 months Treasury Bills rates. All figures are annualized. Columns 2, 3, and 4 display the basic outcome of the mean variance approach. Next columns show information provided by zero investment strategy. The risk is measured by the notional value of the zero investment strategy per monetary unit of the position in the S\&P 500 portfolio (risk level). It expresses the proportion of the amount invested in the S\&P 500 portfolio that would have been absorbed by the cost of hedging it. The risk-adjusted performance has a twofold expression, quantitative and qualitative. The former consists of the notional return and expresses how many risk premium points have been captured per each risk level point. The latter consists of the performance category. It is the consequence of assigning each notional return to its corresponding category according to the four performance levels identified in Section 6 . The last two columns focus on the efficiency in the generation of the notional return through the management of the risk level. The equally weighted level column displays the values of risk premia for which the notional return and the risk level would have had the same impact. The composition ratio indicates the influence of the notional return with respect to the risk level on the risk premium generation, showing how many notional return points stem from each risk level point.

Table 4. S\&P 500 Performance Categories between 1959 and 2013: Summary

POSITIONS BETWEEN 1959 AND 2013

$\begin{array}{ccc}\text { Long hedging } & 31 & 56.36 \% \\ \text { Long } & 10 & 18.18 \% \\ \text { Short } & 8 & 14.55 \% \\ \text { Short hedging } & 6 & 10.91 \% \\ \text { Total } & 55 & 100.00 \%\end{array}$

POSITIONS BETWEEN 1959 AND 1999

$\begin{array}{ccc}\text { Long hedging } & 26 & 63.41 \% \\ \text { Long } & 6 & 14.63 \% \\ \text { Short } & 6 & 14.64 \% \\ \text { Short hedging } & 3 & 7.32 \% \\ & 41 & 100 \%\end{array}$

POSITIONS BETWEEN 2000 AND 2013

$\begin{array}{ccc}\text { Long hedging } & 5 & 35.71 \% \\ \text { Long } & 4 & 28.57 \% \\ \text { Short } & 2 & 14.29 \% \\ \text { Short hedging } & 3 & 21.43 \% \\ \text { Total } & 14 & 100.00 \%\end{array}$

COMPOSITION RATIO BETWEEN 1959 AND 2013

Risk premium return based

Risk premium risk based

Total
55

1

55

Table 4 summarizes the analysis of Table 3: In the period 1959-2013 the predominant performance category has been long hedging ( $56 \%$ of the years), with only 6 years falling into the short hedging category ( $11 \%$ of the years). Nevertheless, if we focus on the 11 years after the burst of the internet bubble followed by the real estate bubble (2000-2013), we find 5 long hedging years (2003, 2006, 2009, 2012, and 2013), counterbalanced by 3 short hedging 
years (2000, 2002, and 2008), 4 long years (2004, 2007, 2010, and 2011) and 2 short years (2001 and 2005). Between 1959 and 1999 we have 26 long hedging years (63.41\%), 6 long years (14.63\%), 6 short years (14.63\%), and 3 short hedging years $(7.32 \%)$. Except in 1969, the risk premium is return based. Figure 3 illustrates this evolution. Its top plot compares the risk premia between 1959 and 2013 with their reference hedging levels. Its bottom plot shows the equivalent evolution of notional returns by comparing their actual values with the reference values +1 and -1 for long hedging and short hedging. From these distributions we can draw the conclusion that the market performance between 1959 and 2013 supports the hypothesis which holds that stocks are the adequate assets for long term investing, although the investor must be able to face the consequences of substantial loses or even very high loses in some periods, which in this table are indicated as short hedging periods. Nevertheless, we can observe that consecutive short and short hedging periods are rare. They only appear in years 1973-1974 (short and short hedging) and 2001-2002 (short hedging, short and short hedging). The hardest short hedging year has been 2008, but it has been followed by a long hedging year (2009), two long ones (2010-2011) and two long hedging years again (2012-2013), although the investor has needed to wait three years to recoup the losses of 2008.

\section{Conclusions}

This paper applies zero-investment strategies built up with options to the analysis of risk premium. Then, the risk premium turns out to be the product of the notional return on the option based zero-investment strategy per the total risk level. The latter coincides with the notional value of the strategy when the initial value of the asset is normalized to 1. The notional return on the zero-investment strategy becomes a risk-adjusted performance measure expressing actual risk premium points per unit of risk level. The notional value is a sole and increasing function of volatility. As a performance measure, the notional return classifies assets automatically into four categories according to the highest position in which they would have produced profits: long hedging, long, short, and short hedging. The composition ratio complements the notional return as a performance measure by showing the weight of the notional return with respect to the risk level in the generation of the risk premium. It enables us to identify if the risk premium is notional return dominated, equally weighted or risk level dominated.

The notional return is quasi-proportional to the Sharpe ratio for the most usual range of volatilities $(5 \%-50 \%)$ with a proportionality constant of 2.5 . This result enables us to place immediately any value of the Sharpe ratio into the four categories we have identified with the limits 0.40 for long hedging and -0.40 for short hedging. The quasi proportionality property basically shows that the notional return and the Sharpe ratio create mutual synergy. The notional return leads to classify performance into the aforementioned four categories, while the Sharpe ratio, once regarded from the perspective of the quasi proportionality, makes possible to identify the corresponding category without calculating the notional return.

The features of notional return make it an appropriate measure for the performance of market indices. Mainly because the notional return contains a benchmark in itself that leads to place the performance of each period into its corresponding category. This means that we classify the performance of the market according to the opportunities of generating notional return embedded in its own risk level that have been effectively turned into risk premium. Besides, the composition ratio adds additional information about the predominance of notional return or risk level into the index risk premium.

To sum up, the contribution of this paper to the existing literature can be summarized in four points: First, the identification of the qualitative performance categories through the notional return. Second, the fact that the qualitative performance can be applied to benchmarks. Third, the identification of the equally weighted level and the composition ratio. The risk premium can be expressed as the product between the notional return and the risk level. The composition ratio, in turn, shows to which extent the contribution of the notional return to the risk premium overperforms or underperforms the one of the risk level. Fourth, the equivalence, for the usual volatility intervals, between the notional return and the Sharpe ratio. This equivalence enables us to express the frontiers in the hedging levels in terms of the Sharpe ratio. The notional return does not substitute the Sharpe ratio, but introduces a new interpretation of it.

\section{Appendix}

Sharpe ratio $(S)$ and notional return may lead to different conclusions. The cause of the contradiction is, simply, the different meaning of Sharpe ratio and notional return. Nevertheless, it can be shown that both ratios are quasi-proportional for the usual range of volatilities, which furnishes a new interpretation of Sharpe ratio.

Let us define the relative risk level $(\ell)$ as the quotient between the risk level and its corresponding volatility. Taking (7) into account, we can write: 


$$
\ell=\frac{\gamma}{\sigma}=\frac{1}{\sigma} \cdot\left[2 \cdot N\left(\frac{\sigma}{2}\right)-1\right]
$$

The relative risk level approaches $1 / \sqrt{2 \pi}$, numerically 0.398942 , when volatility approaches zero, and zero when volatility approaches infinity. It is a concave monotonic decreasing function of volatility.

From (13) and (34) we can write Sharpe ratio as the product of relative risk level by notional return:

$$
S=\ell \zeta
$$

It stems from this result that relative risk level constitutes the link between Sharpe ratio and notional return. On the basis of this relationship, we can show that Sharpe ratio and notional return are quasi-proportional. Let us multiply both sides of (35) by a constant $k$ :

$$
k S=k \ell \zeta
$$

The quasi-proportionality property fulfils for a specific range of volatilities if, for this range, the product between the constant and relative risk level approaches 1 . Then, the product between the constant and Sharpe ratio approaches notional return:

$$
k \ell \rightarrow 1 \Rightarrow k S \rightarrow \zeta
$$

The election of the constant is central in this respect. To obtain a theoretical value for it, we propose to minimize the sum of the square differences between the product $k \ell$ and 1 . We denote by $Y$ the sum of the squares in the volatility interval $[a, b]$ :

$$
Y=\int_{a}^{b}(k \ell-1)^{2} d \sigma
$$

Developing the square, substituting the relative risk level $\ell$ by its value according to (34), and applying some basic properties of integral calculus, we can write:

$$
Y=k^{2} \int_{a}^{b} \frac{\left(2 N\left(\frac{\sigma}{2}\right)-1\right)^{2}}{\sigma^{2}} d \sigma-2 k \int_{a}^{b} \frac{2 N\left(\frac{\sigma}{2}\right)-1}{\sigma} d \sigma+\int_{a}^{b} d \sigma
$$

The first two integrals can be solved through numerical methods. The third is trivial. Deriving (39) with respect to $k$, and equating the derivative to zero, we obtain the expression of the constant that fulfils the property of least squares:

$$
k=\frac{\int_{a}^{b} \frac{2 N\left(\frac{\sigma}{2}\right)-1}{\sigma} d \sigma}{\int_{a}^{b} \frac{\left(2 N\left(\frac{\sigma}{2}\right)-1\right)^{2}}{\sigma^{2}} d \sigma}
$$

Since the second derivative is positive, we have a minimum. Both integrals can be solved through numerical methods. Recalling (7) and (34), we realize that:

$$
k=\frac{\int_{a}^{b} \ell d \sigma}{\int_{a}^{b} \ell^{2} d \sigma}
$$

Table 2 shows the values of $k$ for different volatility ranges. It also shows the products between the constant and relative risk level placed at both extremes of each interval. The maximum positive difference between the approximation $k \cdot \ell$ and notional return takes place for the minimum volatility in the range, while the maximum negative difference happens for the maximum volatility. The small difference we obtain for the usual volatility range $[5 \%, 50 \%]$ and the fact that this difference does not increase significantly for a range such wide as $[0.01 \%, 100 \%]$ prove the robustness of the quasi-proportionality. In effect, this product is confined into the interval [1.0037, 0.9935] for volatility interval $[5 \%, 50 \%]$ and into the interval $[1.0136,0.9702]$ for volatility interval $[0.01 \%, 100 \%]$. Summing up: 


$$
k=\frac{\int_{0.05}^{0.5} \ell \cdot d \sigma}{\int_{0.05}^{0.5} \ell^{2} \cdot d \sigma}=2.51625
$$

Thus, we can substitute the exact relationship between Sharpe ratio and notional return that stems from (35) for the approximate relationship:

$$
S k \approx \zeta
$$

This simplification has a twofold advantage. First, it enables us to regard Sharpe ratio as a change of scale of notional return. Next, it relates Sharpe ratio to the performance levels of notional return. When notional return equates 1 , Sharpe ratio approximately equates the inverse of the constant:

$$
\zeta=1 \Rightarrow S \approx \frac{1}{2.51625}=0.397417
$$

Thus, we can say that a Sharpe ratio over 0.40 means that the asset has overcome its long hedging level, while Sharpe ratios under -0.39 mean that the asset has fallen under its short hedging level.

In order to check the quasi-proportionality for the S\&P 500, following the data displayed in Table 3 we regress notional return against Sharpe ratio, finding that:

$$
\begin{gathered}
\zeta=-0.000477+2.508702 \cdot S \\
t \quad(-1.42) \quad(6443) \\
R^{2}=0.9999
\end{gathered}
$$

which we can take as an empirical confirmation of the quasi-proportionality property.

In addition, the product between the constant and risk level $(\gamma)$ approaches volatility. This property stems from (34) and (37). The former enables to write risk level as the product between relative risk level and volatility. The latter states that the product between the constant and relative risk level approaches 1 . Thus, for volatility ranges for which the quasi-proportionality holds, risk level and volatility produce the same risk rankings.

\section{References}

Aumann, R., \& Serrano, R. (2008). An economic index of riskiness. Journal of Political Economy 116 (5), $810-836$. http://dx.doi.org/10.1086/591947

Bernardo, A. E., \& Ledoit, O. (2000). Gain, loss, and asset pricing. The Journal of Political Economy, 108(1), $144-172$. http://dx.doi.org/10.1086/262114

Bertrand, B., \& Pringent J. (2011). Omega performance measure and portfolio insurance. Journal of Banking \& Finance, 35 (7), 1811-23. http://dx.doi.org/10.1016/j.jbankfin.2010.12.001

Black, F., \& Scholes, M. (1973). The pricing of options and corporate liabilities. Journal of Political Economy, 81 (3), 637-659. http://dx.doi.org/10.1086/260062

Corrado, C. J., \& Su, T. (1996). Skewness and kurtosis in S\&P 500 index returns implied by option prices. The Journal of Financial Research, 19(2), 175-192. http://dx.doi.org/10.1111/j.1475-6803.1996.tb00592.x

Dyk, F. v., Vuuren, G. v., \& Heymans, A. (2014). Hedge fund performance evaluation using the sharpe \& omega ratios. International Business \& Economics Research Journal (Online), 13(3), 485-512.

Eling, M., \& Schuhmacher, F. (2007). Does the choice of performance measure influence the evaluation of hedge funds? Journal of Banking \& Finance 31, (9). 2632-2647. http://dx.doi.org/10.1016/j.jbankfin.2006.09.015

Farinelli S., \& Tibiletti, L. (2008). Sharpe thinking in asset ranking with one-sided measures. European Journal of Operational Research, 185(3), 1542-1547. http://dx.doi.org/10.1016/j.ejor.2006.08.020

Giannotti, C., \& Mattarocci, G. (2013). Risk measurement choice in selecting REITs: Evidence from the U.S. market. Journal of Real Estate Portfolio Management, 19(2), 137-15 3. http://dx.doi.org/10.15396/eres2011_274

Graham, J. R., \& Harvey,C.R. (1997). Grading the performance of market-timing newsletters. Financial Analysts Journal 53, (6), 54-66. http://dx.doi.org/10.2469/faj.v53.n6.2130 
Hentati, R., \& Prigent, J. (2012). Portfolio performance maximization with generalized kappa ratio. Rochester: Social Science Research Network. http://dx.doi.org/10.2139/ssrn.2084930

Homm, U., \& Pigorsch, C. (2012). Beyond the Sharpe ratio: an application of the Aumann-Serrano index to performance measurement. Journal of Banking \& Finance, 36 (8), 2274-2284. http://dx.doi.org/10.1016/j.jbankfin.2012.04.005

Jarrow, R., \& Rudd, A. (1982). Approximate option valuation for arbitrary stochastic processes. Journal of Financial Economics, 10(3), 347-369. http://dx.doi.org/10.1016/0304-405X(82)90007-1

Jensen, M. C. (1968). The performance of mutual funds in the period 1945-1964. Journal of Finance 23, (2) 389-416. http://dx.doi.org/10.1111/j.1540-6261.1968.tb00815.x

Jurczenko, E., Maillet, B., \& Negrea, B. (2004). A note on skewness \& kurtosis adjusted option pricing models under the martingale restriction. Quantitative Finance, 4 (5), 479-488. http://dx.doi.org/10.1080/14697680400020309

Kazemi, H., Schneeweis, T., \&. Gupta, R. (2004). Omega as a performance measure. Journal of Performance Measurement, 8, 3, 16-25.

Modigliani, F., \& Modigliani L. (1997). Risk-adjusted performance. Journal of Portfolio Management, 23 (2), 45-54. http://dx.doi.org/10.3905/jpm.23.2.45

Proelss, J., \& Schweizer, D. (2014). Polynomial goal programming \& the implicit higher moment preferences of US institutional investors in hedge funds. Financial Markets \& Portfolio Management, 28(1), 1-28. http://dx.doi.org/10.1007/s11408-013-0221-x

Rachev, S.T., Stoyanov, S., \& Fabozzi, F.J. (2007). Advanced Stochastic Models, Risk Assessment, \& Portfolio Optimization: The Ideal Risk, Uncertainty, \& Performance Measures. New York: John Wiley.

Shadwick, W.F. \& Keating C. (2002). A universal performance measure. Journal of Performance Measurement, 6 (3), 59-84.

Sharpe, W. F. (1966). Mutual fund performance.The Journal of Business (Pre-1986), 39(1), 119-138. http://dx.doi.org/10.1086/294846

Sharpe, W.F. (1994) Sharpe ratio. Journal of Portfolio Management, 21 (1), 49-88. http://dx.doi.org/10.3905/jpm.1994.409501

Sortino, F. A. \& Satchell S. E. ( 2001). Managing downside risk in financial markets. Oxford: Butterworth-Heinemann.

Sortino, F. A., \& van der Meer, R. (1991). Downside risk. Journal of Portfolio Management, 17(4), 27-31. http://dx.doi.org/10.3905/jpm.1991.409343

Treynor, J. L. (1965). How to Rate Management of Investment Funds. Harvard Business Review, 43, 63-75.

Treynor, J. L., \& Black, F. (1973). How to use security analysis to improve portfolio selection. Journal of Business 46, (1), 66-86. http://dx.doi.org/10.1086/295508

\section{Notes}

Note 1. Equation (7) is obtained applying the Black and Scholes formula to a case in which the present value of the strike price equates the underlying asset value, taking a maturity of one period, and, next, dividing the result by the value of the underlying asset. Since the equality between the present value of the strike price and the underlying asset equates at the same time the call and put values, it is indifferent to use Black and Scholes for a call or a put. If options mature at $n$ periods, we have to substitute $\sigma$ by $\sigma \sqrt{n}$. This simply means to replace volatility at one period by its equivalent at $n$ periods.

Note 2. To this end the models by Jarrow and Rudd (1982) or Corrado and Su (1996), modified by Jurczenko, Maillet and Negrea (2004), can be applied. 